\title{
Comparison of long-term survival and toxicity of simultaneous integrated boost vs conventional fractionation with intensity-modulated radiotherapy for the treatment of nasopharyngeal carcinoma
}

\author{
This article was published in the following Dove Press journal: \\ OncoTargets and Therapy \\ 31 March 2016 \\ Number of times this article has been viewed
}

\section{Hengmin Tao ${ }^{1,2}$ \\ Yumei Wei' \\ Wei Huang' \\ Xiujuan Gai ${ }^{1,2}$ \\ Baosheng $\mathrm{Li}^{\prime}$}

'Department of 6th Radiation Oncology, Shandong Cancer Hospital and Institute, ${ }^{2}$ School of Medicine and Life Sciences, Jinan UniversityShandong Academy of Medical Sciences, Jinan, People's Republic of China
Correspondence: Baosheng Li Department of 6th Radiation Oncology, Shandong Cancer Hospital and Institute, No 440, Jiyan Road, Huaiyin District, 250 I I 7 Jinan, Shandong, People's Republic of China $\mathrm{Tel}+8653167626162$ Email baoshli1963@163.com
Aim: In recent years, the intensity-modulated radiotherapy with simultaneous integrated boost (IMRT-SIB) and intensity-modulated radiotherapy with conventional fractionation (IMRT-CF) have been involved in the treatment of nasopharyngeal carcinoma (NPC). However, the potential clinical effects and toxicities are still controversial.

Methods: Here, 107 patients with biopsy-proven locally advanced NPC between March 2004 and January 2011 were enrolled in the retrospective study. Among them, 54 patients received IMRT-SIB, and 53 patients received IMRT-CF. Subsequently, overall survival (OS), 5-year progression-free survival (PFS), 5-year locoregional recurrence-free survival (LRFS), and relevant toxicities were analyzed.

Results: In the present study, all patients completed the treatment, and the overall median follow-up time was 80 months (range: $8-126$ months). The 5-year OS analysis revealed no significant difference between the IMRT-SIB and IMRT-CF groups ( $80.9 \%$ vs $80.5 \%, P=0.568)$. In addition, there were also no significant between-group differences in 5-year PFS (73.3\% vs $74.4 \%, P=0.773)$ and 5 -year LRFS (88.1\% vs 90.8\%, $P=0.903)$. Notably, the dose to critical organs (spinal cord, brainstem, and parotid gland) in patients treated by IMRT-CF was significantly lower than that in patients treated by IMRT-SIB (all $P<0.05$ ).

Conclusion: Both IMRT-SIB and IMRT-CF techniques are effective in treating locally advanced NPC, with similar OS, PFS, and LRFS. However, IMRT-CF has more advantages than IMRT-SIB in protecting spinal cord, brainstem, and parotid gland from acute and late toxicities, such as xerostomia. Further prospective study is warranted to confirm our findings.

Keywords: intensity-modulated radiotherapy with simultaneous integrated boost, intensitymodulated radiotherapy with conventional fractionation, nasopharyngeal carcinoma, survival, toxicities

\section{Introduction}

Nasopharyngeal carcinoma (NPC) is one of the most common head and neck tumors in the People's Republic of China. The incidence of NPC was $>20 / 100,000$ in southern China. Most of the patients with NPC are usually diagnosed in locally advanced, nonmetastatic stage III or IV. ${ }^{1,2}$ To date, radiotherapy (RT) has been recommended as the first option for treatment of NPC. Thus, it is essential to investigate multimodal RT to improve survival status of patients with NPC.

With the advent of RT equipment and computer technology, intensity-modulated radiotherapy (IMRT) has been extensively utilized in the treatment of NPC due to its 
benefits in accurately targeting organs, reducing toxicities of organs at risk, and enhancing dose escalation. Importantly, the dosimetric advantage of IMRT has been widely identified and betters local control rate. ${ }^{3}$ On the other hand, simultaneous integrated boost (SIB), also called simultaneous modulated and accelerated RT, can deliver different doses to target regions according to the level of risk, which exploited the "dose-painting" capacity of IMRT. ${ }^{4}$ The safety and efficacy of IMRT-SIB have been confirmed in patients with NPC. ${ }^{5-7}$ Meanwhile, IMRT with conventional fractionation (IMRT-CF) has also been widely used in the treatment of NPC; however, the efficacy of IMRT-CF was little reported, in that CF (2.0 Gy/ fraction) was subjected to two-dimensional conventional radiotherapy (2D-RT) or three-dimensional conventional radiotherapy (3D-RT). Besides, few studies compared the efficacy and safety of IMRT-SIB and IMRT-CF in recent decades.

Therefore, in the present study, we enrolled 107 patients with biopsy-proven and locally advanced NPC, and analyzed overall survival (OS), 5-year progression-free survival (PFS), 5-year locoregional recurrence-free survival (LRFS), and relevant toxicities. Our study will contribute to the improvement of clinical IMRT treatment strategies in patients with NPC.

\section{Methods}

\section{Patients}

The present retrospective cohort was composed of 107 newly diagnosed and previously untreated patients with histopathologically confirmed NPC. All 107 patients were treated with IMRT in Shandong Cancer Hospital and Institute between March 2004 and January 2011. Median age of all patients was 43 years (16-78 years). Human participant approval was obtained from the ethical committee of Shandong Cancer Hospital and Institute. Written informed consent from patients was obtained. Routine workup included a thorough physical examination, hematologic and biochemistry profiles, fiberoptic endoscope examination of the nasopharynx, and magnetic resonance imaging (MRI) or contrast-enhanced computed tomography (CT) of the head and neck, with which the status of the primary tumor and regional lymph nodes could be accurately evaluated. At the same time, the chest $\mathrm{X}$-ray or CT, whole-body bone scan, and abdominal region ultrasonography were used to exclude distant metastasis. Staging of the patients was completed using the American Joint Committee on Cancer/Union for International Cancer Control 2002 system for staging classification of NPC.

\section{Radiotherapy}

All patients were immobilized with a thermoplastic mask. The simulation CT images extended from the vertex of the skull to $5 \mathrm{~cm}$ inferior of the clavicular heads, and were obtained at a slice thickness of $3 \mathrm{~mm}$. All patients were treated by IMRT.

In the IMRT-SIB group, the gross tumor volume (GTV) included the primary nasopharyngeal tumor and lymph nodesinvolved tumors demonstrated by contrast enhancement CT or MRI. Clinical target volume (CTV) 1, a high-risk region, was defined by appending a $5 \mathrm{~mm}$ margin to GTV, which included the inferior sphenoid sinus, clivus, skull base, nasopharynx, ipsilateral parapharyngeal space, posterior third of the nasal cavity, maxillary sinuses, and Level II, III, and Va lymph nodes. CTV2, a low-risk region, was the lower neck below the cricothyroid membrane. A smaller margin $(3 \mathrm{~mm})$ was also acceptable in these regions close to the critical structures, such as brainstem, optic nerves, and optic chiasm. CTV intermediate- and low-risk regions were contoured according to Radiation Therapy Oncology Group (RTOG) recommendations. Planning target volume (PTV) was defined by adding a $5 \mathrm{~mm}$ margin to the CTV in all dimensions. The prescribed dose was $66 \mathrm{~Gy} / 30$ fractions at $2.2 \mathrm{~Gy} /$ fraction to the planning gross tumor volume (PGTV), $60 \mathrm{~Gy} / 30$ fractions at $2.0 \mathrm{~Gy} /$ fraction to the PTV1, and $54 \mathrm{~Gy} / 30$ fractions at $1.8 \mathrm{~Gy} /$ fraction to the PTV2. Total radiation doses of 66-74, 60, and 54 Gy were delivered to PGTV, PTV1, and PTV2, respectively, with 30-34 fractions at five fractions per week.

In the IMRT-CF group, CTV included the primary nasopharyngeal tumor, lymph nodes-involved tumor, the high-risk regions including the entire nasopharynx, skull base, clivus, inferior sphenoid sinus, retropharyngeal lymph nodal regions, pterygoid fossae, parapharyngeal space, the posterior third of the nasal cavity, maxillary sinuses, and any high- or low-risk nodal regions including bilateral cervical lymph nodes Level II-V. PTV was generated with $5 \mathrm{~mm}$ margin. PTV was delivered at $2 \mathrm{~Gy} /$ fraction for 25 fractions with total dose of $50 \mathrm{~Gy}$, followed by twice replanning. The first and second replanning were generated, respectively, using a "shrinking-field technique" after 25 fractions and 30 fractions. The accumulated radiation doses were 70-74 Gy to PTV of the GTV for the primary nasopharyngeal tumors and the involved lymph nodes, 60 Gy to high-risk PTV, and 50 Gy to low-risk PTV. All patients were treated with one fraction daily for 5 days per week. The target prescription dose and the critical structures limit dose were planned according to the RTOG trial 0225 criteria. The irradiated doses of both IMRT techniques are summarized in Table 1.

\section{Chemotherapy}

In this study, the chemotherapy regimens were cisplatin alone, cisplatin/docetaxel, and cisplatin/5-fluorouracil. These 
Table I Dosimetric difference of both IMRT strategies

\begin{tabular}{lll}
\hline Technique & $\begin{array}{l}\text { BED (tumor) } \\
\left(\mathbf{G y}_{10}\right)\end{array}$ & $\begin{array}{l}\text { BED (late) } \\
\left(\mathbf{G y}_{10}\right)\end{array}$ \\
\hline IMRT-SIB $(2$-phase) & & \\
PGTV $2.2 \times 30+2.0 \times 4=74$ Gy & 90.12 & 128.27 \\
PTV-HR 2.0×30=60 Gy & 72.00 & 100.00 \\
PTV-LR I.8×30=54 Gy & 63.72 & 86.40 \\
IMRT-CF $(3-$ phase) & & \\
PGTV 2.0×25+2.0×5+2.0×7=74 Gy & 88.80 & 123.33 \\
PTV-HR 2.0×25+2.0×5=60 Gy & 72.00 & 100.00 \\
PTV-LR 2.0×25=50 Gy & 60.00 & 83.33 \\
\hline
\end{tabular}

Notes: BED calculation is based on the linear-quadratic model for cell kill with an $\alpha / \beta$ of 10 for tumors and an $\alpha / \beta$ of 3 for late-responding normal tissue. The BED is calculated by the following equation: $\mathrm{BED}=n d[1+d /(\alpha / \beta)]$.

Abbreviations: IMRT, intensity-modulated radiotherapy; BED, biological effective dose; IMRT-SIB, IMRT with simultaneous integrated boost; PGTV, planning gross tumor volume; PTV-HR, high-risk planning target volume; PTV-LR, low-risk planning target volume; IMRT-CF, IMRT with conventional fractionation.

chemotherapy regimens were known to possess similar activity and effectiveness for treatment of NPC, and were administered as neoadjuvant, concurrent, or adjuvant treatment (Table 2).

\section{Follow-up}

The duration of follow-up was calculated from the first day of treatment to either the day of death or day of the last

Table 2 Clinical characteristics and baseline levels between IMRT-SIB and IMRT-CF

\begin{tabular}{|c|c|c|c|}
\hline Characteristics & $\begin{array}{l}\text { IMRT-SIB } \\
(n=54)\end{array}$ & $\begin{array}{l}\text { IMRT-CF } \\
(n=53)\end{array}$ & $P$-value \\
\hline Sex & & & 0.874 \\
\hline Male & 39 & 39 & \\
\hline Female & 15 & 14 & \\
\hline Age (years) & & & 0.147 \\
\hline$\leq 45$ & 31 & 23 & \\
\hline$>45$ & 23 & 30 & \\
\hline T classification & & & 0.95 \\
\hline TI & 3 & 4 & \\
\hline $\mathrm{T} 2$ & 13 & 13 & \\
\hline T3 & 29 & 26 & \\
\hline $\mathrm{T} 4$ & 9 & 10 & \\
\hline $\mathrm{N}$ classification & & & 0.84 \\
\hline No & 7 & 5 & \\
\hline $\mathrm{NI}$ & 7 & 9 & \\
\hline N2 & 31 & 32 & \\
\hline N3 & 9 & 7 & \\
\hline Staging & & & 0.917 \\
\hline II & 3 & 2 & \\
\hline III & 33 & 34 & \\
\hline IVA & 9 & 10 & \\
\hline IVB & 9 & 7 & \\
\hline \multicolumn{4}{|l|}{ Chemotherapy } \\
\hline Neoadjuvant & 13 & 14 & 0.780 \\
\hline $\mathrm{DDP}+5-\mathrm{FU}$ & 7 & 6 & \\
\hline Docetaxel + DDP & 6 & 8 & \\
\hline Concurrent & 54 & 53 & 0.874 \\
\hline DDP & 54 & 53 & \\
\hline
\end{tabular}

(Continued)
Table 2 (Continued)

\begin{tabular}{clll}
\hline Characteristics & $\begin{array}{l}\text { IMRT-SIB } \\
(\mathbf{n}=\mathbf{5 4})\end{array}$ & $\begin{array}{l}\text { IMRT-CF } \\
(\mathbf{n}=\mathbf{5 3})\end{array}$ & P-value \\
\hline Adjuvant & 39 & 38 & 0.952 \\
$\quad$ DDP + 5-FU & 17 & 19 & \\
$\quad \begin{array}{l}\text { Docetaxel + DDP } \\
\text { RT duration (days) }\end{array}$ & 22 & 19 & \\
Mean \pm SD & $45.74 \pm 2.21$ & $50.74 \pm 2.53$ & $<0.001$ \\
\hline
\end{tabular}

Abbreviations: IMRT-SIB, intensity-modulated radiotherapy with simultaneous integrated boost; IMRT-CF, intensity-modulated radiotherapy with conventional fractionation; DDP, cisplatin; 5-FU, 5-fluorouracil; RT, radiotherapy; SD, standard deviation; $\mathrm{T}$, tumor; $\mathrm{N}$, node.

follow-up. Patients were interviewed and examined at least every 3 months during the first 2 years, and subsequent every 6 months. Follow-up information must include clinical examination, and CT or MRI of the head and neck region. When patients had potential locoregional recurrence or distant metastasis, additional examinations or imaging modalities were performed to confirm disease progression at the discretion of the treating physician. Missing data were completed by calling the patient or the treating physician. Acute and late toxicities were scored according to the Common Terminology Criteria for Adverse Events version 3.0. Besides, the diagnostic criteria for injury of the nervous system involves the following: lesions or necrosis in the nervous system showed contrast enhancement on postcontrast T1-weighted MRI, heterogeneous hyperintense on T2-weighted MRI, and homogeneous peri-necrosis hyperintense on T2-weighted MRI. Furthermore, the recurrence or metastasis of tumor must be excluded when determining the site of radiation encephalopathy.

Last, the time to the first defined event OS, PFS, and LRFS was assessed. OS was measured from the first day of treatment until death or follow-up deadline. PFS was measured from the first day of treatment to the date of the first observation of local or regional recurrence or distant metastasis. LRFS was measured from the date of treatment to the date of the first observation of local and regional recurrence.

\section{Statistical methods}

All statistical analyses were performed using SPSS 17.0 software (SPSS Inc., IBM Company, Armonk, NY, USA). The chi-square test was used to calculate statistical group comparisons of categorical variables. Survival analysis was performed using the Kaplan-Meier method, and comparisons were calculated using the log-rank test. The Mann-Whitney test and log-rank method were used to estimate the differences in both groups. Multivariate analyses with the Cox proportional hazards model were used to test independent prognostic factor by backward elimination of insignificant 
explanatory variables. The Cox proportional hazards model was used to calculate hazard ratios. $P<0.05$ was considered statistically significant, and all $P$-values correspond to twosided significance tests.

\section{Results}

\section{Patient characteristics and baseline level}

To figure out the baseline level of all patients, we summarized the characteristics of patients and treatment modality. As shown in Table 2, 78 males and 29 females were included, and the male/female ratio was calculated as $\sim 2.6: 1$. The most patients presented with stage III (62.6\%) and stage IV (32.7\%). According to statistics, there were no significant between-group differences in sex, age, $\mathrm{T}$ and $\mathrm{N}$ classifications, and clinical stage (all $P>0.05$ ), indicating that our study was reliable.

\section{Dosimetric comparison}

As shown in Table 3, we summarized the maximal doses $\left(D_{\max }\right)$ to the critical structures (spinal cord, brainstem, crystalline lens, and optic nerve), and calculated the mean doses $\left(D_{\text {mean }}\right)$ to the parotid glands. Our results revealed that the doses of spinal cord, brainstem, and parotid gland were significantly lower in patients treated by IMRT-CF than in those patients treated by IMRT-SIB (all $P<0.05$ ).

Figure $1 \mathrm{~A}-\mathrm{C}$ illustrates the deformable image registration process in IMRT-SIB group. After recontouring parotids on 30th fraction images, both parotid volumes were mapped to the initial treatment plan with the same beam configurations. These plans were referred to as "simulated plans", and demonstrated significant migration of the parotids, especially toward the high-dose PTV regions, which would result in increased dose to the parotids. In addition, the dose-volume histograms showed that estimated delivered doses to both parotids were higher than initial plans during the RT course (Figure 2).

\section{Radiation toxicities}

To elucidate the potential toxicities of IMRT-SIB and IMRT-CF, we summarized all types of toxicities in our study, and toxicities after 6 months following RT were defined as late radiation toxicities. No treatment-related deaths were observed in either cohort. Acute and late radiation toxicities are shown in Table 4. Systemic acute toxicities were similar in both groups.

Of 107 patients, the most common late toxicities included xerostomia and hearing loss, which were observed in $40.1 \%$ and $30.8 \%$ of patients, respectively. Briefly, in the IMRTSIB group, xerostomia can be found in 26 patients (48.1\%), whereas the IMRT-CF group had 17 patients $(32.1 \%)$ with xerostomia; the difference was significant $(P<0.001)$. Accordingly, the rate of late hearing loss in the IMRT-SIB group was also greater than that in the IMRT-CF group; however, no significant statistic difference was observed $(P=0.491)$.

Subsequently, we summarized late toxicities of the nervous system, and found that in IMRT-SIB group, three patients with NPC (5.5\%) suffered from late toxicities of the nervous system, including two patients (1.87\%) with temporal lobe injury and one patient $(0.93 \%)$ with brainstem injury. In addition to that, no injury was observed in temporal lobe, brainstem, and spinal cord.

\section{Patterns of failure and survival outcome}

In the present study, the overall median follow-up time was 80 months (range: 8-126 months). Of 107 patients,

Table 3 Adverse event grade according to Common Terminology Criteria for Adverse Events version 3.0

\begin{tabular}{|c|c|c|c|c|c|c|c|c|c|c|c|}
\hline \multirow[t]{2}{*}{ Toxicity } & \multicolumn{5}{|c|}{ IMRT-SIB } & \multicolumn{5}{|c|}{ IMRT-CF } & \multirow[t]{2}{*}{$P$-value } \\
\hline & 0 & $\mathbf{I}$ & 2 & 3 & 4 & 0 & $\mathbf{I}$ & 2 & 3 & 4 & \\
\hline \multicolumn{12}{|l|}{ Acute toxicities } \\
\hline Dematitis & 0 & 23 & 27 & 4 & 0 & 0 & 21 & 30 & 2 & 0 & 0.636 \\
\hline Mucositis & 0 & 21 & 14 & 19 & 0 & 0 & 20 & 19 & 14 & 0 & 0.465 \\
\hline Xerostomia & 10 & 21 & 23 & 0 & 0 & 13 & 21 & 19 & 0 & 0 & 0.683 \\
\hline Dysphagia & 20 & 26 & 6 & 2 & 0 & 23 & 25 & 4 & I & 0 & 0.813 \\
\hline Hearing loss & 34 & 19 & I & 0 & 0 & 36 & 16 & I & 0 & 0 & 0.859 \\
\hline \multicolumn{12}{|l|}{ Late toxicities } \\
\hline Xerostomia & 28 & 9 & 15 & 2 & 0 & 36 & 6 & II & 0 & 0 & $<0.001$ \\
\hline Temporal lobe injury & 52 & I & I & 0 & 0 & 53 & 0 & 0 & 0 & 0 & 0.368 \\
\hline Brainstem injury & 53 & I & 0 & 0 & 0 & 53 & 0 & 0 & 0 & 0 & 0.32 \\
\hline Trismus & 53 & 1 & 0 & 0 & 0 & 53 & 0 & 0 & 0 & 0 & 0.375 \\
\hline Hearing loss & 33 & 15 & 6 & 0 & 0 & 41 & 9 & 3 & 0 & 0 & 0.314 \\
\hline
\end{tabular}

Abbreviations: IMRT-SIB, intensity-modulated radiotherapy with simultaneous integrated boost; IMRT-CF, intensity-modulated radiotherapy with conventional fractionation. 


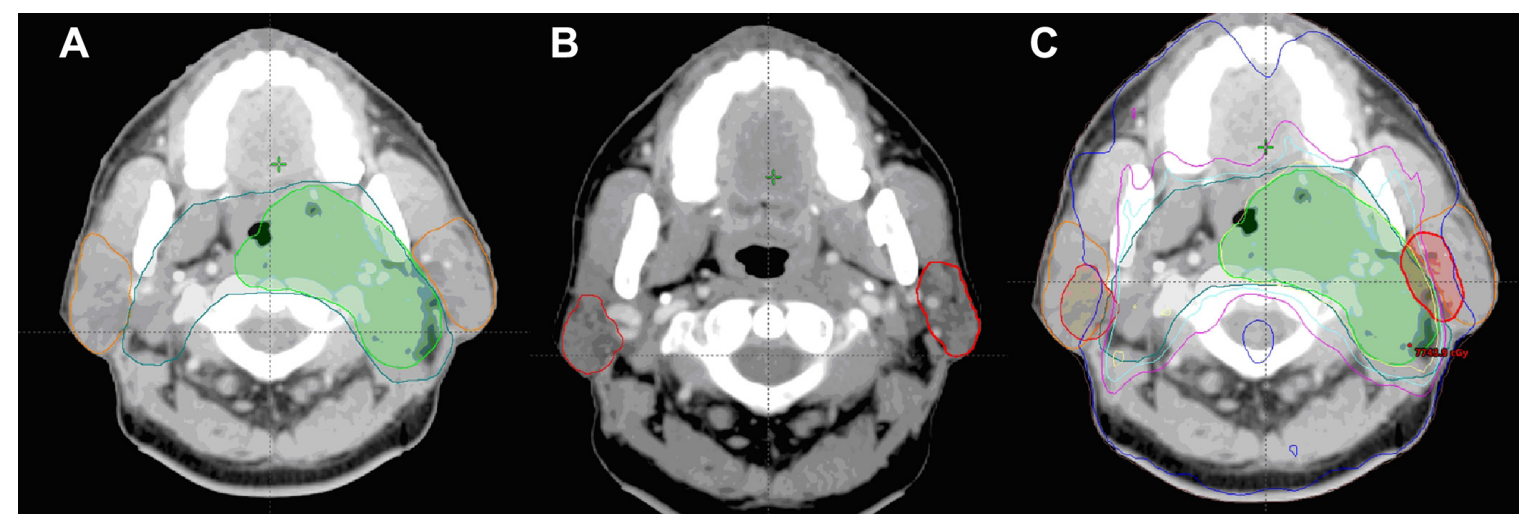

Figure I Axial CT images of one patient with target volume and parotid gland contours in IMRT-SIB group.

Notes: (A) Manually drawn target volume (green and blue) and parotid gland (orange) contours on first planning CT. (B) Manually drawn parotid gland contours (red) on second planning CT. (C) The deformable image registration of $(\mathbf{A})$ and $(\mathbf{B})$.

Abbreviations: CT, computed tomography; IMRT-SIB, intensity-modulated radiotherapy with simultaneous integrated boost.

17 patients $(15.8 \%)$ developed locoregional relapse, 22 patients $(20.5 \%)$ presented with distant metastasis, and 30 patients $(28 \%)$ were dead. Seventeen patients with locoregional relapse included eight patients $(7.5 \%)$ with local relapse, seven patients $(6.5 \%)$ with regional relapse, and the rest of two patients $(1.9 \%)$ with both local and regional relapse. Besides, two patients (1.9\%) developed both locoregional and distant failure.

Based on follow-up data, the overall 5-year OS, PFS, and LRFS rates were $80.7 \%, 72.9 \%$, and $89.4 \%$, respectively. According to statistics, we found no significant betweengroup differences in 5-year OS $(80.9 \%$ vs $80.5 \%, P=0.568)$ (Figure 3), PFS (73.3\% vs $74.4 \%, P=0.773$ ) (Figure 4), and
LRFS (88.1\% vs $90.8 \%, P=0.903)$ (Figure 5). These results indicated that both IMRT-SIB and IMRT-CF can benefit patients and improve the survival status.

\section{Prognostic factors}

To throw light on the independent prognostic factor, we conducted multivariate analysis using variables in this study, including age ( $>45$ years vs $\leq 45$ years), $\mathrm{T}$ classification (T1-2 vs T3-4), N classification (N0-1 vs N2-3), and RT method (IMRT-CF vs IMRT-SIB). All these variables were summarized and calculated in the Cox proportional hazards model using the backward elimination method. Multivariate analysis revealed that age and $\mathrm{N}$ classification act as

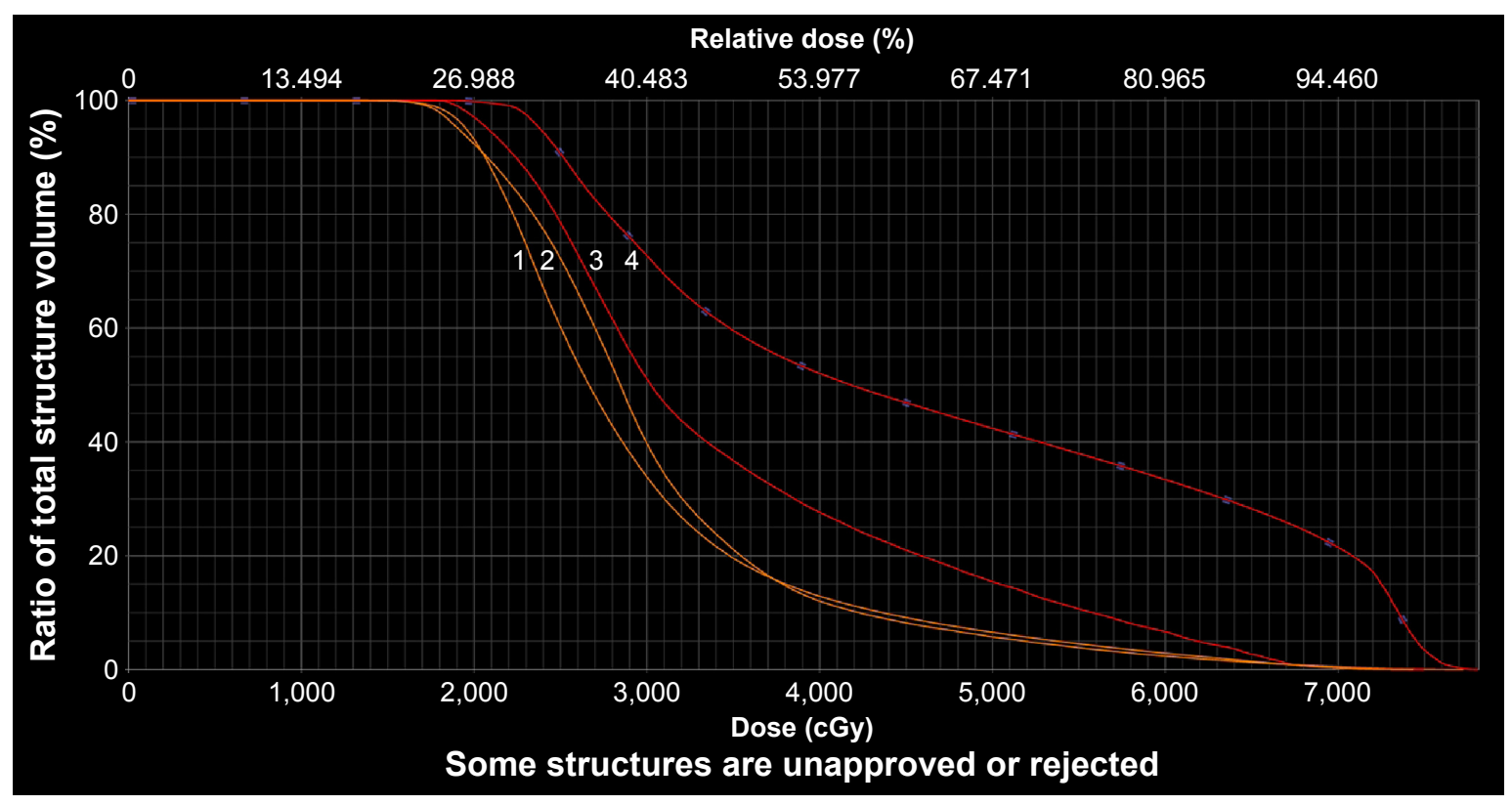

Figure 2 The dose-volume histograms of the parotids.

Notes: I: the right parotid of first planning CT. 2: the left parotid of first planning CT. 3: the right parotid of second planning CT. 4: the left parotid of second planning CT. Abbreviation: CT, computed tomography. 
Table 4 Comparison of parameters in IMRT-SIB and IMRT-CF groups

\begin{tabular}{|c|c|c|c|c|}
\hline$\overline{\text { OAR }}$ & Parameter & IMRT-SIB & IMRT-CF & $\overline{P \text {-value }}$ \\
\hline Spinal cord & $D_{\max }(G y)$ & $45.93 \pm 4.20$ & $43.19 \pm 2.40$ & $<0.00$ I \\
\hline Brainstem & $D_{\max }(G y)$ & $56.80 \pm 3.16$ & $53.91 \pm 4.19$ & $<0.001$ \\
\hline Left crystalline lens & $D_{\max }(G y)$ & $5.65 \pm 1.26$ & $5.27 \pm 1.25$ & 0.174 \\
\hline Right crystalline lens & $D_{\max }(\mathrm{Gy})$ & $5.59 \pm 1.05$ & $5.44 \pm 1.21$ & 0.360 \\
\hline Left optic nerve & $D_{\max }(\mathrm{Gy})$ & $28.30 \pm 16.75$ & $22.80 \pm 15.06$ & $0.08 I$ \\
\hline Right optic nerve & $D_{\max }(G y)$ & $27.23 \pm 16.52$ & $21.99 \pm 15.95$ & 0.063 \\
\hline Left parotid & $D_{\text {mean }}(G y)$ & $32.99 \pm 2.66$ & $30.35 \pm 2.25$ & $<0.00 \mathrm{I}$ \\
\hline Right parotid & $D_{\text {mean }}(G y)$ & $32.76 \pm 2.75$ & $30.03 \pm 2.36$ & $<0.00 \mathrm{I}$ \\
\hline
\end{tabular}

Note: IMRT-SIB and IMRT-CF data presented as mean \pm standard deviation.

Abbreviations: IMRT-SIB, intensity-modulated radiotherapy with simultaneous integrated boost; IMRT-CF, intensity-modulated radiotherapy with conventional fractionation; OAR, organs at risk; $D_{\max }$, maximal dose to the volume; $D_{\text {mean }}$, mean dose to the volume.

significant predictive factors for $\operatorname{OS}(P<0.001$ and $P=0.039$, respectively), $\mathrm{PFS}(P=0.003$ and $P=0.009$, respectively), and LRFS ( $P=0.011$ and $P=0.031$, respectively). However, RT method cannot serve as a significant prognostic factor for OS, PFS, and LRFS. At the same time, it should be noted that $\mathrm{T}$ classification was another prognostic factor only for PFS $(P=0.037)$ (Table 5).

\section{Discussion}

In recent decades, IMRT has been widely utilized and benefited most patients with NPC in short-term treatment. It is reported that IMRT has some advantages in improving the local control rate and quality of life. ${ }^{8-10}$ Till now, IMRT has been developed into two models, like IMRT-CF and IMRTSIB. However, the real effects of IMRT-CF and IMRT-SIB on NPC treatment were not well featured. In the present study, the rate of 2-year OS and PFS in IMRT-CF-treated

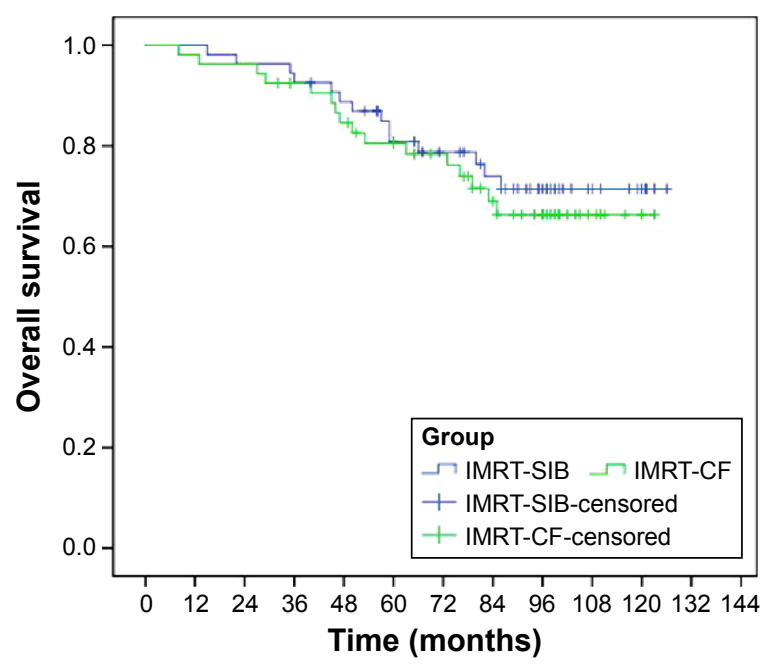

Figure 3 Comparison of overall survival between patients treated with IMRT-SIB and those treated with IMRT-CF $(P=0.568)$.

Abbreviations: IMRT-SIB, intensity-modulated radiotherapy with simultaneous integrated boost; IMRT-CF, intensity-modulated radiotherapy with conventional fractionation. patients with NPC was $96.2 \%$ and $94.4 \%$, respectively. Consistent with our data, $\mathrm{Ng}$ et al included 193 patients in their trial, and concluded that 2-year OS and PFS in IMRT-CF-treated patients with NPC increased up to $92 \%$ and $95 \%$, respectively. ${ }^{8}$ As reported, IMRT-SIB has been widely investigated in NPC treatment with a nominal total dose of 64.8-76 Gy to GTV in 2.12-2.4 Gy/fraction over 27-35 fractions. ${ }^{8,11,12}$ Overall, NPC patients with IMRT-SIB treatment achieved a local or locoregional control rate of $88 \%-96 \%$ based on $2-5$ years of follow-up, though SIB dose fractionation varied in these studies. Our results showed that the rate of 5-year OS, PFS, and LRFS in patients with IMRTSIB treatment was $80.9 \%, 73.3 \%$, and $88.1 \%$, respectively. In accordance to our data, Sun et al ${ }^{9}$ conducted a retrospective analysis on 868 nonmetastatic patients with NPC treated by IMRT-SIB, and found that the rate of 5-year LRFS and PFS for all patients was $91.8 \%$ and $77.0 \%$, respectively, and the

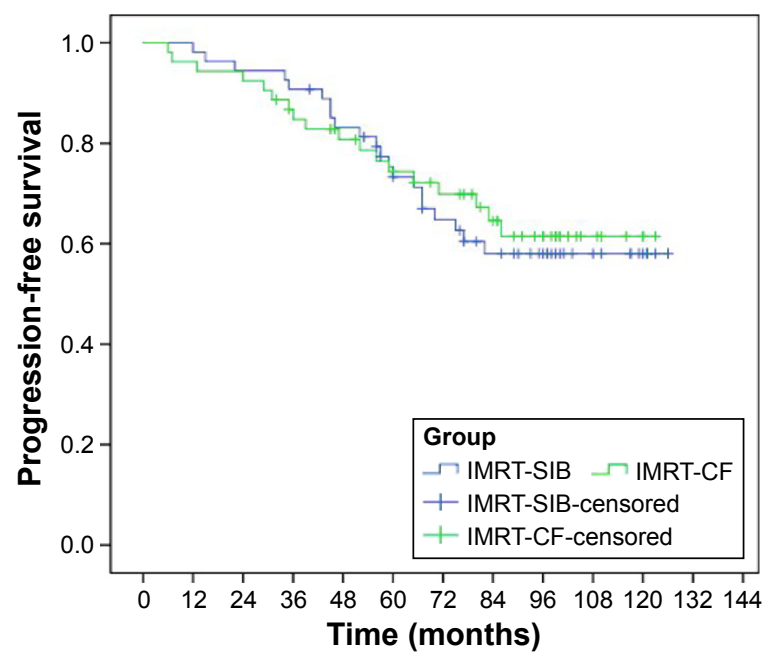

Figure 4 Comparison of progression-free survival between patients treated with IMRT-SIB and those treated with IMRT-CF $(P=0.773)$.

Abbreviations: IMRT-SIB, intensity-modulated radiotherapy with simultaneous integrated boost; IMRT-CF, intensity-modulated radiotherapy with conventional fractionation. 


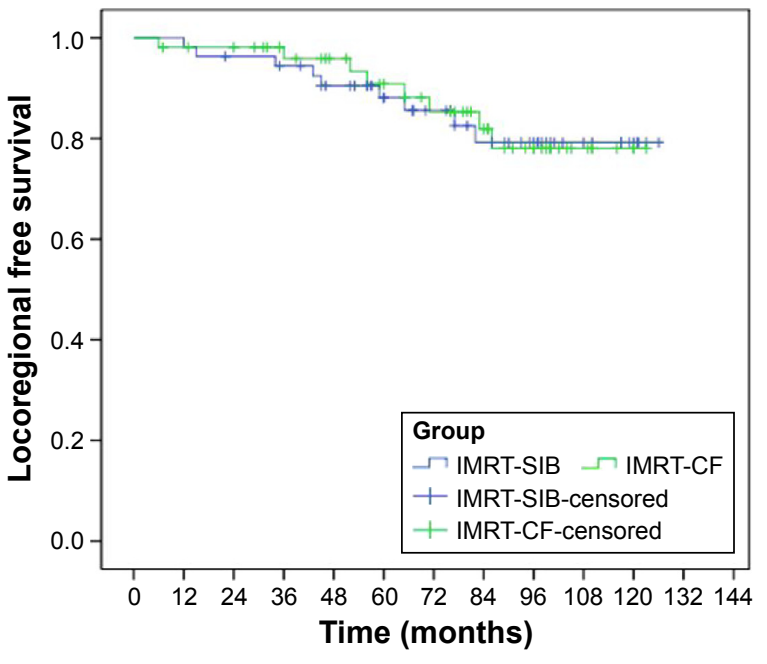

Figure 5 Comparison of locoregional recurrence-free survival between patients treated with IMRT-SIB and those treated with IMRT-CF $(P=0.903)$.

Abbreviations: IMRT-SIB, intensity-modulated radiotherapy with simultaneous integrated boost; IMRT-CF, intensity-modulated radiotherapy with conventional fractionation.

5 -year OS rate was up to $83.2 \%$. These results indicated that both IMRT-SIB and IMRT-CF can benefit patients and improve the survival status.

Chen et $\mathrm{al}^{13}$ and Nesrin et $\mathrm{al}^{14}$ performed dosimetric studies comparing the target volume coverage and normal tissue sparing of IMRT-SIB vs IMRT-CF for NPC. Both the groups found that the maximal dose to spinal cord and brainstem was lower in IMRT-CF than in IMRT-SIB. They suggested that normal tissues embedded within the target regions may receive higher doses per fraction compared to the doses given by IMRT-CF delivery techniques. Therefore, IMRT-CF may be more appropriate than IMRT-SIB when the dose given to the normal tissues is the major concern. This is the same direction in our study which showed that IMRT-CF provided better sparing of spinal cord and brainstem than did the IMRT-SIB ( $P=0.001$ for spinal cord and $P=0.001$ for brainstem). This indicates that a small area of brainstem and spinal cord, especially in T3-T4 patients, was inclined to receive a high dose per fraction (2.2 Gy/fraction) when patients were subjected to IMRT-SIB due to its approach to the boost volume. Therefore, caution should be taken when applying IMRT-SIB technique when these critical structures are very close to the boost volume. ${ }^{13}$

On the other hand, reports by Chen et $\mathrm{al}^{13}$ and Nesrin et $\mathrm{al}^{14}$ demonstrated that IMRT-SIB could provide better sparing of the parotid glands than IMRT-CF. However, our study identified that IMRT-CF provided better sparing of parotid glands in comparison with IMRT-SIB. This is probably related to the following two reasons: (1) CT scans were performed after the 25th fraction in IMRT-CF, while after 30th in IMRT-SIB group, and then the treatment plans were re-conducted according to these scans. With the shrinking of the primary tumor and nodal masses, the parotids might deviate to the GTV (lymph node involvement) (Figure 1C), which results in higher actual dose in parotid gland than expected (Figure 2). Zhang et al ${ }^{15}$ reported that there was a benefit to bilateral parotids through replanning at the fifth week. (2) If the involved lymph nodes in Level II were very large and close to parotids, the calculated total dose to the parotid gland may increase.

Afterward, we analyzed radiation-induced toxicities. Of 107 patients, the most common late toxicities included xerostomia and hearing loss, which were observed in $40.1 \%$ and $30.8 \%$ of patients, respectively. Consistent with our results, Peng et $\mathrm{al}^{16}$ showed that $39.5 \%$ of patients with NPC had grade I-II xerostomia. Moreover, our results also showed that patients receiving IMRT-CF had a lower incidence of acute and late xerostomia than those who received IMRT-SIB. We think that it may be attributed to the statistical difference in the mean dose to parotid gland. ${ }^{15}$ Recently, Marzi et al ${ }^{17}$ reported a minor increase of parotid $D_{\text {mean }}$ based on the tolerance dose that can result in potential severe xerostomia. Obviously, IMRT-CF significantly decreased the doses to parotids (Table 3 ) to reduce xerostomia-related symptoms and improve the quality of life. At the same time, an insignificant high incidence of grade 3 dermatitis, oropharyngeal mucositis, dysphagia, and late hearing loss in the IMRT-SIB group was also observed in this work.

Table 5 Multivariate analysis of OS, PFS, and LRFS

\begin{tabular}{|c|c|c|c|c|c|c|}
\hline Factor & $\begin{array}{l}\text { OS hazard ratio } \\
(95 \% \mathrm{Cl})\end{array}$ & $P$-value & $\begin{array}{l}\text { PFS hazard ratio } \\
(95 \% \mathrm{Cl})\end{array}$ & $P$-value & $\begin{array}{l}\text { LRFS hazard ratio } \\
(95 \% \mathrm{Cl})\end{array}$ & $P$-value \\
\hline Age $>45$ years vs $\leq 45$ years & $1.055(1.024-1.087)$ & $<0.001$ & $1.039(1.013-1.067)$ & 0.003 & $1.070(1.016-1.128)$ & 0.011 \\
\hline T classification & $0.043(0.165-1.123)$ & 0.085 & $0.439(0.202-0.953)$ & 0.037 & $0.717(0.174-2.960)$ & 0.646 \\
\hline $\mathrm{N}$ classification & $2.568(1.049-6.286)$ & 0.039 & $3.000(1.323-6.802)$ & 0.009 & $9.342(1.227-71.126)$ & 0.031 \\
\hline Radiotherapy (IMRT-CF vs IMRT-SIB) & $1.378(0.477-3.98 \mathrm{I})$ & 0.554 & $0.685(0.322-1.456)$ & 0.325 & $0.697(0.179-2.714)$ & 0.603 \\
\hline
\end{tabular}

Note: $P$-values were calculated using an adjusted Cox proportional hazards regression model.

Abbreviations: OS, overall survival; $\mathrm{Cl}$, confidence interval; PFS, progress-free survival; LRFS, locoregional recurrence-free survival; IMRT-CF, intensity-modulated radiotherapy with conventional fractionation; IMRT-SIB, intensity-modulated radiotherapy with simultaneous integrated boost; T, tumor; N, node. 
Finally, we demonstrated that $\mathrm{N}$ staging and age act as a significant predictive factor for OS, PFS, and LRFS. However, $T$ stage was not a significant prognostic factor in this study. In contrast, previous studies showed that both $\mathrm{T}$ and $\mathrm{N}$ categories act as a significantly independent factor. ${ }^{8,9,18}$ Maybe, our sample size in this study is relatively small. Most importantly, our analysis revealed that RT was not a prognostic factor for OS, PFS, and LRFS, and the difference between IMRT-SIB and IMRT-CF did not affect survival outcome.

In the clinical practice, the overall radiotherapy treatment time of IMRT-CF was significantly longer than IMRT-SIB. In this study, although a lengthening of overall radiotherapy treatment time of 5 days was obtained with the IMRT-CF technique, IMRT-CF only adds three times the cost for the RT. Besides, due to acute IMRT-SIB-related toxicities, the corresponding cost of supportive care and management will increase. Joiner ${ }^{19}$ reported that reduction in overall treatment time was assumed to reduce the risk of tumor colognes regrowth during the late phase of radiation treatment, and improve the probability of tumor control. In our study, the biological effective dose to PGTV (90.12 $\mathrm{Gy}_{10}$ ) was higher in IMRT-SIB than in IMRT-CF $\left(88.80 \mathrm{~Gy}_{10}\right)$. However, the overall 5-year LRFS rates were not significantly different between the two groups $(88.1 \%$ vs $90.8 \%, P=0.903$ ).

To our knowledge, this is the first single-institution study to investigate the difference between IMRT-CF and IMRTSIB in patients with NPC. The main limitations in this study exist in retrospective property and relatively small sample size. To draw a definitive conclusion, a randomized Phase II study on IMRT-SIB than IMRT-CF in patients with locally advanced NPC is being conducted in our hospital. This study will allow for the standardization of IMRT technique for patients with NPC.

\section{Conclusion}

The IMRT-SIB and IMRT-CF can benefit patients with local advanced-stage NPC, and better the survival status. The IMRT-CF has more advantages than IMRT-SIB in protecting spinal cord, brainstem, and parotid gland from acute and late toxicities, such as xerostomia. Thus, IMRT-CF should be recommended for patients with locally advanced NPC. The results of this study will be further validated in prospective, multicenter controlled trials.

\section{Acknowledgments}

This study was supported by the Natural Science Foundation of Shandong Province, People's Republic of China (Grant
No ZR2014YL034) and by Taishan Scholars Program of Shandong Province, People's Republic of China (Grant No ts20120505).

\section{Disclosure}

The authors report no conflicts of interest in this work.

\section{References}

1. Jemal A, Bray F, Center MM, Ferlay J, Ward E, Forman D. Global cancer statistics. CA Cancer J Clin. 2011;61(2):69-90.

2. Chen L, Mao YP, Xie FY, et al. The seventh edition of the UICC/AJCC staging system for nasopharyngeal carcinoma is prognostically useful for patients treated with intensity-modulated radiotherapy from an endemic area in China. Radiat Oncol Biol Phys. 2012;104(3):331-337.

3. Lai SZ, Li WF, Chen L, et al. How does intensity-modulated radiotherapy versus conventional two-dimensional radiotherapy influence the treatment results in nasopharyngeal carcinoma patients? Int J Radiat Oncol Biol Phys. 2011;80(3):661-668

4. Butler EB, Teh BS, Grant WH 3rd, et al. Smart (simultaneous modulated accelerated radiation therapy) boost: a new accelerated fractionation schedule for the treatment of head and neck cancer with intensity modulated radiotherapy. Int J Radiat Oncol Biol Phys. 1999;45(1):21-32.

5. Lee N, Harris J, Garden AS, et al. Intensity-modulated radiation therapy with or without chemotherapy for nasopharyngeal carcinoma: radiation therapy oncology group phase II trial 0225. J Clin Oncol. 2009; 7(22):3684-3690.

6. Kwong DL, Sham JS, Leung LH, et al. Preliminary results of radiation dose escalation for locally advanced nasopharyngeal carcinoma. Int J Radiat Oncol Biol Phys. 2006;64(2):374-381.

7. Xiao WW, Huang SM, Han F, et al. Local control, survival, and late toxicities of locally advanced nasopharyngeal carcinoma treated by simultaneous modulated accelerated radiotherapy combined with cisplatin concurrent chemotherapy: long-term results of a phase 2 study. Cancer. 2011;117(9):1874-1883.

8. Ng WT, Lee MC, Hung WM, et al. Clinical outcomes and patterns of failure after intensity-modulated radiotherapy for nasopharyngeal carcinoma. Int J Radiat Oncol Biol Phys. 2011;79(2):420-428.

9. Sun X, Su S, Chen C, et al. Long-term outcomes of intensity-modulated radiotherapy for 868 patients with nasopharyngeal carcinoma: an analysis of survival and treatment toxicities. Radiother Oncol. 2014; 110(3):398-403.

10. Tao CJ, Lin L, Zhou GQ, et al. Comparison of long-term survival and toxicity of cisplatin delivered weekly versus every three weeks concurrently with intensity-modulated radiotherapy in nasopharyngeal carcinoma. PLoS One. 2014;9(10):E110765.

11. Bakst RL, Lee N, Pfister DG, et al. Hypofractionated dose-painting intensity modulated radiation therapy with chemotherapy for nasopharyngeal carcinoma: a prospective trial. Int J Radiat Oncol Biol Phys. 2011;80(1):148-153.

12. Wong FC, Ng AW, Lee VH, et al. Whole-field simultaneous integratedboost intensity-modulated radiotherapy for patients with nasopharyngeal carcinoma. Int J Radiat Oncol Biol Phys. 2010;76(1):138-145.

13. Chen SW, Yang SN, Liang JA, et al. Comparative dosimetric study of two strategies of intensity-modulated radiotherapy in nasopharyngeal cancer. Med Dosim. 2005;30(4):219-227.

14. Nesrin D, Stephen K, Bahman E, et al. Assessment of different IMRT boost delivery methods on target coverage and normal-tissue sparing. Int J Radiat Oncol Biol Phys. 2003;57(5):1480-1491.

15. Zhang X, Li M, Cao J, et al. Dosimetric variations of target volumes and organs at risk in nasopharyngeal carcinoma intensity-modulated radiotherapy. Br J Radiol. 2012;85(1016):e506-e513.

16. Peng G, Wang T, Yang KY, et al. A prospective, randomized study comparing outcomes and toxicities of intensity-modulated radiotherapy vs. conventional two-dimensional radiotherapy for the treatment of nasopharyngeal carcinoma. Radiother Oncol. 2012;104(3):286-293. 
17. Marzi S, Pinnarò P, D'Alessio D, et al. Anatomical and dose changes of gross tumour volume and parotid glands for head and neck cancer patients during intensity-modulated radiotherapy: effect on the probability of xerostomia incidence. Clin Oncol. 2012;24(3):e54-e62.

18. Lee AW, Sze WM, Au JS, et al. Treatment results for nasopharygeal cacinoma in the modern era: the Hong Kong experience. Int J Radiat Oncol Biol Phys. 2005;61(4):1107-1116.
19. Joiner MC. Hyperfractionation and Accelerated Radiotherapy. London: Arnold; 1997:123-131.

\section{Publish your work in this journal}

OncoTargets and Therapy is an international, peer-reviewed, open access journal focusing on the pathological basis of all cancers, potential targets for therapy and treatment protocols employed to improve the management of cancer patients. The journal also focuses on the impact of management programs and new therapeutic agents and protocols on

\section{Dovepress}

patient perspectives such as quality of life, adherence and satisfaction. The manuscript management system is completely online and includes a very quick and fair peer-review system, which is all easy to use. Visit http://www.dovepress.com/testimonials.php to read real quotes from published authors.

Submit your manuscript here: http://www.dovepress.com/oncotargets-and-therapy-journal 